\title{
Movilizaciones de LAS JUVENTUdes en Honduras: LA EXPERIENCIA DE LOS ESTUDIANTES UNIVERSITARIOS DE LA UNAH (2009-2017)
}

\author{
Youth Mobilization in Honduras: The Experience of University Students at the UNAH (2009-2017)
}

\author{
Pablo A. Vommaro \\ Galel Briceño-Cerrato
}

Resumen: En la última década, las juventudes fueron protagonistas de movilizaciones y conflictos sociales en América Latina y el mundo. En este artículo se analizan las dinámicas del Movimiento Estudiantil Universitario en Honduras, en el marco de los procesos de resistencia juveniles en los países del Triángulo Norte centroamericano, con enfoque en las juventudes inscritas en la sede de Tegucigalpa de la Universidad Nacional Autónoma de Honduras. Planteamos que las resistencias juveniles desbordan el espacio universitario y se expresan con propuestas creativas e innovadoras frente a la represión gubernamental. El artículo se basa en revisión de prensa gráfica y digital, en bibliografía secundaria y en observaciones participantes y no participantes en eventos de protesta sucedidos en Honduras entre 2009 y 2017.

Palabras clave: jóvenes, movimientos estudiantiles universitarios, resistencias, participación política.

Abstract: In recent years, young people have been protagonists of mobilizations and social conflicts in Latin America and the world. This paper analyzes the dynamics of mobilization by the university student movement in Honduras, in the context of resistance by young people in the Central American "Northern Triangle" countries. We focus on young students enrolled at the National Autonomous University of Honduras, particularly at the Tegucigalpa campus. The authors consider that youth resistance goes beyond university campuses and finds expression through creative and innovative proposals, even as students face government repression. This paper is based on a review of graphic and digital press reports, analysis of secondary bibliography, and participant and non-participant observations at protests that took place in Honduras between 2009 and 2017.

Keywords: youth, university student movement, resistance, political participation.

Pablo A. Vommaro. Doctor en Ciencias Sociales por la Universidad de Buenos Aires, Argentina. Profesor de Historia de la UBA. Investigador del CONICET. Co-coordinador del Grupo de Estudios de Políticas y Juventudes (GEPoJu) en el Instituto Gino Germani de la Universidad de Buenos Aires, Argentina. Docente de la Facultad de Filosofía y Letras de la Universidad de Buenos Aires, Argentina. Temas de especialización: historia latinoamericana contemporánea, procesos de participación juvenil y políticas públicas. Correo electrónico: pvommaro@gmail.com
Galel Briceño Cerrato. Sociólogo por la Universidad Nacional Autónoma de Honduras. Estudiante de maestría en Gobierno y Especialización en Estudios Políticos de la Universidad de Buenos Aires, Argentina. Temas de especialización: estudios políticos, procesos de gobierno y políticas públicas. Correo electrónico: galelbriceo@gmail.com.

Enviado a dictamen: 31 de octubre de 2017

Aprobación: 18 de mayo de 2018

Revisiones: 1 
Gobierno y condiciones de vida de las juventudes en Honduras

L a gestión del gobierno de Honduras a cargo de Juan Orlando Hernández (2014-2018) tiene características autoritarias que pueden analizarse como continuación de regímenes dictatoriales impulsados por las élites políticas en los años setenta y ochenta, que adquirieron una nueva forma a partir del golpe de Estado de 2009. ${ }^{1}$

De esta manera, la historia reciente de Honduras ha experimentado cambios políticos sustanciales asociados al achicamiento del Estado que han derivado en la reducción de las funciones sociales de éste vinculadas a derechos de salud, educación, justicia o trabajo, y en la ampliación de las atribuciones represivas y policiales. Esto ha llevado a abruptos procesos de desestructuración institucional, a un aumento de la inseguridad, al asesinato de líderes sociales a manos de grupos paraestatales y al deterioro de la calidad de vida de la mayoría de la población. Estos cambios tendientes a la degradación institucional y social han implicado la privatización y la patrimonialización (Wiarda, 1997; Castro-Gómez, 2010: 44)² de las instituciones públicas, así como la imposición de un modelo económico y social excluyente y segregador que necesita de la represión para imponerse sobre la población (Alcántara e Ibeas, 2001). Estas políticas regresivas de degradación de derechos, retracción de funciones del Estado y aumento de la represión se despliegan con más fuerza entre algunos grupos sociales. Aquí pondremos el foco en la situación de las juventudes, particularmente de las que viven en el conglomerado urbano de Tegucigalpa.

La gestión gubernamental de imposición del ya mencionado modelo excluyente y segregador en Honduras ha conllevado al agravamiento de las desigualdades sociales. Según datos del Banco Mundial para 2016: "cerca del 66\% de la población vive en la pobreza. En zonas rurales aproximadamente uno de cada 5 hondureños vive en pobreza extrema o con menos de US\$1.90 al día" (Banco Mundial, 2017: 1). Esta inequidad en la distribución de recursos afecta de manera directa y particularmente sensible a los grupos sociales más jóvenes, lo que hace necesario incorporar la dimensión generacional al análisis de las desigualdades. ${ }^{3}$

En efecto, según cifras coincidentes de diversos organismos internacionales y nacionales (PNUD, 2015; CEPAL y UNFPA, 2014; INE, 2017), la pobreza juvenil en Honduras alcanza al 62\% o 63\% de esta población, ${ }^{4}$ y son casi la mitad los jóvenes que, teniendo trabajo, están en condiciones precarias y de subempleo. Asimismo, Honduras es no sólo uno de los países más pobres de América Latina, sino uno de los más desiguales, con un índice de Gini superior al 0.50, que en muchos períodos roza el 0.60 (INE, 2017: 1).

Por otra parte, en Honduras "el 68\% de población es menor de 30 años" (Pena, 2014: 1). Según las tendencias demográficas arrojadas por el Instituto Nacional de Estadística, la población joven, además de ser mayoría, tiende a ascender en número en los próximos años (INE, 2017: 19). Esta situación fue denominada "bono demográfico" por diversos organismos internacionales (PNUD, 2015; CEPAL y UNFPA, 2014), los cuales pusieron el foco en la oportunidad que significaba tener una creciente población juvenil en un futuro próximo - y no avejentada o estancada como en otros paísespara la implementación de políticas de desarrollo y la mejora de las condiciones de vida de la sociedad. Sin embargo, todo indica que esta oportunidad se desaprovechó en Honduras, ya que los gobiernos desde 2009 no promovieron políticas públicas de juventud que posibilitaran una mejora en la calidad de vida de este grupo poblacional y oportunidades de participación y compromiso social. Al contrario, como se muestra en este artículo, las juventudes hondureñas aparecen como más empobrecidas, más desiguales y más reprimidas en sus posibilidades de participación en el espacio social.

En efecto, Honduras tiene una de las tasas de homicidios más altas del istmo Centroamericano, la cual oscila entre setenta y noventa personas asesinadas por cada cien mil habitantes entre 2014 y 2016 Asimismo, la violencia adquiere dimensiones políticas por los asesinatos de líderes y referentes sociales, en su mayoría jóvenes, por parte de grupos paraestatales. En este sentido, el Observatorio de la Violencia de la Universidad Nacional Autónoma de Honduras (OV- 
UNAH) apunta que en 2015 murieron 361 personas en 98 masacres - homicidios de más de dos personas simultáneamente-

En el mismo sentido, el Instituto Universitario de Democracia, Paz y Seguridad (IUDPAS, 2016) señala que desde enero de 2010 hasta marzo de 2016 se registraron 1183 homicidios de estudiantes en todo el país. Desagregando estos datos, se evidencia que 160 de los asesinados eran estudiantes de educación superior, 624 de secundaria, 124 de primaria, y 275 víctimas fueron identificadas como estudiantes sin determinar el nivel educativo al que pertenecían. 2014 fue el año en el que más muertes violentas de estudiantes se registraron, con 254 casos. Los más afectados por estos homicidios fueron los estudiantes de secundaria al representar el $52.7 \%$ del total, seguidos por los universitarios con el 13.5\%. Como hipótesis de trabajo a desarrollar en otros textos, planteamos que esta diferencia en el conjunto de asesinatos puede deberse a cuestiones vinculadas con procesos etarios de participación en redes de actividades en conflicto con la ley a nivel territorial, y no tanto a procesos específicamente políticos.

Según los análisis realizados, y coincidiendo con diferentes autores hondureños (Sosa, 2013 y 2014), estos asesinatos pueden enmarcarse en el proceso de criminalización de la protesta estudiantil contra las políticas gubernamentales y, entre 2016 y 2017, también contra las de las autoridades de la Universidad Nacional Autónoma de Honduras (UNAH). ${ }^{5}$ Considerando la magnitud y focalización de estos asesinatos y de las políticas represivas y persecutorias, también pueden relacionarse con las nociones de juvenicidio (Valenzuela, 2015: 20) y aniquilamiento simbólico y físico (Nateras, 2015: 102) que diversos autores mexicanos han elaborado para interpretar estos procesos a nivel regional. ${ }^{6}$

A partir de lo dicho, puede entenderse la importancia de analizar los procesos sociales y políticos que viven las juventudes hondureñas. En este trabajo nos enfocaremos en los estudiantes universitarios ya que constituyeron uno de los grupos sociales más visibles en las movilizaciones y conflictos políticos desarrollados luego de 2009. En particular, abordaremos la experiencia del Movimiento Estudiantil Universitario (MEU), organización que surgió como Movimiento Amplio Universitario (MAU) tras el golpe de Estado de 2009 y que cambió su nombre al calor de las movilizaciones callejeras y los conflictos universitarios que se suscitaron después de la asunción de Juan Orlando Hernández como presidente de Honduras en 2013

El artículo se basa en una revisión de prensa gráfica y digital, en lecturas de bibliografía secundaria y en observaciones participantes y no participantes que los autores realizaron en eventos de protesta sucedidos en Tegucigalpa entre 2014 y 2017.

\section{Participación política juvenil y movimiento estudiantil universitario: aproximaciones y discusiones}

Las discusiones teóricas y empíricas acerca de los vínculos entre juventudes y políticas signaron los estudios de juventudes durante las últimas dos décadas en América Latina. Así, los diagnósticos de efervescencia o de apatía, o bien de novedades, alternativas y retornos, se suceden y superponen en los trabajos académicos y en los debates públicos. Para avanzar en estas cuestiones creemos necesario abordar las transformaciones que experimentó la política en años recientes y explorar la diversidad de prácticas, de formas organizativas y asociativas, de construcciones identitarias y culturales y de modalidades de subjetivación política que produjeron los jóvenes en sus experiencias de participación (Vommaro, 2015a).

Los lugares y las formas de la política tienen diversos modos de expresarse y resolverse: las instituciones político-estatales y representativas son unas, como así también los movimientos sociales, en tanto modalidades y colectivos que, por fuera de la institucionalidad estatal instituida, persiguen objetivos públicos y construyen modos de disputar dirección y gobierno (Tapia, 2008). Por otra parte, coincidimos con Jelin en que lo político no es un a priori o una esencia: diferentes contenidos -incluso algunos considerados tradicionalmente privados o íntimos- pueden asumir 
carácter público y confrontativo y, así, politizarse (Jelin, 1989). Podríamos avanzar aún más y sostener que algunas prácticas culturales juveniles — aun cuando no han sido concebidas como políticas por los actores que las protagonizan - han sido leídas como modos de expresión de politicidad, en tanto "modos de contestar al orden vigente y formas de insertarse socialmente" (Reguillo, 2003), o bien de intervenir en el espacio de "lo común" (Núñez, 2013). Así, prácticas como el arte callejero, determinados consumos culturales y algunas expresiones en el marco de las denominadas culturas juveniles han revelado, para algunos investigadores, un carácter político.

A partir de estos análisis, proponemos el abordaje desde la noción de politización ya que permite considerar el proceso de ampliación de las fronteras de lo político que se produjo en América Latina en los últimos cuarenta años (Vommaro, 2015b). En efecto, la politización de las relaciones y de los espacios cotidianos diluyó ciertas fronteras entre lo privado y lo público, lo que produjo un avance de lo público en tanto producción de lo común y territorio de la política. Desde esta mirada, la política es una producción relacional y dinámica, en proceso; y los jóvenes son protagonistas fundamentales en estas transformaciones de las formas de la política, con sus innovaciones y continuidades respecto a modalidades anteriores (Vommaro, 2013).

Ahora bien, aunque sostenemos que las formas de expresión, producción y práctica de la política pueden multiplicarse y que existen diferentes modos de intervenir en y producir lo público, y aunque partimos de que la política en los jóvenes excede lo instituido, también asumimos que es necesario precisar en qué momentos y situaciones una práctica, una experiencia o una organización se politizan, es decir, adquieren carácter público, conflictivo y colectivo. En este sentido, creemos que la politicidad, o la dimensión política de una práctica o producción, es más una hipótesis, un punto de llegada, que un supuesto de partida. Así, pensamos la politicidad también en términos de la potencialidad política que pueden conllevar las prácticas culturales juveniles. Retomamos entonces las propuestas que realizaron
Bonvillani, Palermo, Vázquez y Vommaro, quienes consideraron que:

[...] la politización es un potencial u horizonte constitutivo de cualquier vínculo social. Sin embargo, para atribuirle carácter político a un colectivo y a un sistema de prácticas sociales, consideramos que es preciso reconocer, al menos, cuatro aspectos: 1) que se produzca a partir de la organización colectiva; 2) que tenga un grado de visibilidad pública (ya sea de un sujeto, de una acción o de una demanda); 3) que reconozca un antagonista a partir del cual la organización adquiere el potencial político; 4) que se formule una demanda o reclamo que adquiera un carácter público y contencioso (Bonvillani et al., 2010).

A partir de lo dicho, sostenemos que es posible observar entre los jóvenes de Honduras y de la región un desplazamiento desde las formas clásicas de organización y participación política, hacia otro tipo de espacios y prácticas en los que no sólo no rechazan la política, sino que se politizan sobre la base de la impugnación de los mecanismos delegativos de participación y toma de decisiones. Éste es el movimiento que signa los procesos de movilización estudiantil que se analizan en este artículo.

En definitiva, proponemos distanciarnos de las miradas esencialistas de la juventud y pensar a los jóvenes como sujetos activos y potentes para, de este modo, analizar sus posibilidades y sus producciones contextualizadas. De esta manera, elaboramos una perspectiva centrada en su protagonismo político en el marco de las particulares condiciones sociohistóricas que experimentan y en las que han intervenido de determinadas maneras para producir sus mundos de vida (Schütz, 1993).

Al concebir las juventudes a partir del enfoque generacional, y al resaltar su dimensión relacional, es ineludible asumir también el vínculo que se establece con otras generaciones, es decir, las dinámicas intergeneracionales. De este modo, asumiendo la diversidad de lo político, pero planteando una 
hipótesis de delimitación e identificación de los procesos de politización, aquí mostramos algunas de las características y formas en las que los jóvenes estudiantes protagonizaron procesos de politización y a partir de los cuales intervinieron en las dinámicas del conflicto social en Honduras, tomando como centro las movilizaciones producidas en la sede de la UNAH en Tegucigalpa.

De esta manera, frente a la crisis política abierta en Honduras luego del golpe de 2009, y tras las posteriores elecciones en las que se sospechó de fraude y manipulación, se han gestado descontentos y olas de movilización juvenil. Estas insurgencias han contemplado en su repertorio de demandas temas como el cese de la violencia estatal y paraestatal, la creación de oportunidades de empleo y educación para todos los jóvenes, la destitución del presidente electo en comicios que se sospecharon fraudulentos y manipulados, la democratización de los espacios de representación política y el esclarecimiento de los actos de corrupción cometidos por el gobierno nacional. Así, los grupos juveniles movilizados, especialmente del movimiento estudiantil universitario, se constituyeron en protagonistas de las principales acciones callejeras y conflictos de los últimos años.

En efecto, los años posteriores a 2009 han estado marcados por olas de movilización estudiantil en la UNAH que han tenido repercusiones en la dinámica política general y han desbordado los espacios universitarios.

\section{La Universidad como territorio de disputa: las movilizaciones estudiantiles como expresión de formas de participación política juvenil}

Como vimos en otros trabajos (Vommaro, 2013, 2015a y 2015b), en la última década se han producido numerosas movilizaciones en América Latina que dinamizaron conflictos sociales y políticos, en muchos casos aún abiertos. En la mayoría de estos procesos de movilización los jóvenes fueron protagonistas activos impulsando organizaciones, movilizándose y ocupando espacios públicos de muchas de las principales ciudades latinoamericanas. Estos procesos de movilización y organización juvenil expresan los rasgos más importantes que delinean las ya mencionadas configuraciones de la política en la región. Así, considerar las formas de participación juvenil es fundamental para comprender las características, dinámicas y sentidos de los procesos políticos nacionales y regionales.

Este proceso latinoamericano se enmarca además en un fenómeno más global que permite identificar que en las primeras décadas del siglo XXI se han producido en diversas regiones del mundo - África del Norte, América Latina, Europa, América del Nortemovilizaciones sociales que tienen a los jóvenes como sus principales protagonistas. Los movimientos de carácter más sociopolítico, como los de la denominada Primavera Árabe que contribuyeron a la caída de distintos gobiernos en África del Norte, los múltiples colectivos que se agrupan bajo la denominación de "indignados" en Europa —sobre todo en España- y Estados Unidos, las organizaciones estudiantiles que luchan por la democratización y la mejora de la calidad de una educación mercantilizada y degradada en América Latina - Chile, Colombia, México, Honduras, Guatemala-, y los jóvenes urbanos movilizados en Brasil, han sido los más visibles en este aspecto, pero no los únicos. Existen también colectivos de indígenas, de trabajadores precarizados, de minorías sexuales, de migrantes, de campesinos, centros culturales o grupos de arte, expresión y comunicación, entre muchos otros, que son activos protagonistas de conflictos y movilizaciones en sus territorios de acción específicos. Los jóvenes de los sectores populares y las periferias de muchas grandes ciudades también han construido colectivos y asociaciones que expresan sus formas singulares de participación y compromiso con lo público y con la transformación de la realidad en la que viven. En muchas de estas organizaciones las disputas territoriales constituyen su principal modalidad de acción y despliegue de iniciativas (Vommaro, 2013: 127-128).

La capacidad organizativa, la visibilidad pública y el renovado interés de muchos jóvenes de la región en la participación política y el compromiso con las cuestiones públicas configuran una coyuntura 
que Ernesto Rodríguez caracteriza como la de los "nuevos movimientos juveniles latinoamericanos" con características más propositivas que reactivas (Rodríguez, 2012)

En los procesos de movilización y organización juvenil que se desplegaron en la América Latina reciente, los colectivos estudiantiles han ocupado un lugar destacado.

Este hecho produjo un regreso de los estudios acerca de los movimientos estudiantiles secundarios y universitarios, que eran considerados fenómenos del pasado y habían perdido importancia frente a formas supuestamente novedosas de expresión juvenil ligadas a lo cultural, a lo estético o a experiencias territoriales o políticas alternativas (Vommaro, 2013 y 2015a).

En efecto, la vida en la universidad y la constitución del movimiento estudiantil han sido puntos de partida obligados para los estudios sobre jóvenes, juventudes y sus relaciones con la política. En América Latina esto cobró una significación especial a partir del movimiento conocido como Reforma Universitaria, iniciado en Córdoba (Argentina) en 1918. La Reforma se extendió rápidamente a otras regiones de Argentina y a otros países de América Latina, y tuvo efectos políticos que excedieron ampliamente al movimiento estudiantil en países como Perú, Chile, México y Cuba (Tünnermann, 2008). ${ }^{7}$

En los movilizados años sesenta y setenta del siglo XX, hablar de juventudes y políticas era hablar de movimiento estudiantil, sobre todo universitario. Así, las obras académicas y los discursos públicos producidos en esos años en general no se refieren al sujeto juvenil como a un actor social de relevancia. Más bien, los jóvenes aparecían solapados detrás de otras filiaciones que se consideraban más importantes y explicativas como la clase social o la condición de estudiante. También se vislumbraban tras algunas producciones o discursos culturales como el rock o movimientos como los hippies, ciertas vanguardias artísticas o asociados a la militancia política, en general partidaria, y también dentro de los grupos armados o guerrillas. Si bien estos espacios estaban mayoritariamente conformados por jóvenes, lo juvenil no era problematizado como tal y la identificación estudiantil prevalecía en aquellos años (Bonvillani et al., 2010).

Por otra parte, los trabajos sobre el movimiento estudiantil en Centroamérica y Honduras han sido poco frecuentes, incluso después de la activación de la movilización juvenil luego de 2009 y de las acciones de los "Camisetas Negras Anti JOH" en 2013. Es como si lo que sucediera en la Universidad no fuera central para comprender las realidades de las prácticas y producciones políticas juveniles en la actualidad hondureña. Ésta es una de las premisas desde las que proponemos comenzar a indagar en este artículo.

A diferencia de la escasez de estudios acerca del movimiento estudiantil universitario en Honduras, en otras regiones de América Latina se han realizado desde 2010 muchas y buenas investigaciones sobre los movimientos estudiantiles secundarios y universitarios.

Un recorrido panorámico por las principales experiencias de politización ${ }^{9}$ estudiantil que se despliegan en América Latina en la actualidad muestra que se trata de organizaciones que se movilizan para expresar posibilidades políticas de establecimiento de relaciones intergeneracionales, a la vez que tienden puentes entre las movilizaciones de los jóvenes y las de otros movimientos y expresiones sociales colectivas más o menos organizadas. Así, se observa como estas movilizaciones superan ampliamente los límites sectoriales - y aun los generacionales - para convertirse en procesos que dinamizan diversas luchas sociales más amplias y expresan impugnaciones al sistema dominante que exceden las cuestiones educativas (Vommaro, 2015a).

Estas experiencias de politización y radicalización juveniles, que desbordan los reclamos sectoriales, pueden analizarse también desde los planteamientos de Badiou (2000), quien sostiene que no se puede "llamar movimiento a aquello que es una simple defensa de un interés", porque "no hay movimiento si solo se trata de una reivindicación particular o interesada". Y agrega que en un movimiento "siempre hay demandas, hay reivindicaciones, hay pedidos", pero el movimiento es "mucho más que esos pedidos, que esas demandas" (Badiou, 2000: 27). En esta clave, 
un movimiento social se constituye como tal cuando es capaz de superar la dimensión sectorial y particular, para expresar aspiraciones políticas más generales que interpelan lo común.

En Honduras, gran parte de losintereses de los grupos dominantes a nivel económico y político están fijados en la educación superior. Esto se puso en evidencia en la injerencia de los últimos gobiernos sobre las decisiones y trasformaciones en materia de política educativa ejecutadas por las autoridades universitarias. De igual manera, el botín de la universidad también pretende ser compartido por grupos corporativos privados. El "modelo de centralización de capital en grupos corporativos privados se ha permeado en el sistema de Educación Superior Pública, en abrupta imposición y sobrevaloración de las instituciones corporativas privadas que venden servicios educativos" (Briceño Cerrato, 2017: 3). Estos procesos de imposición tienen incidencia directa en la organización administrativa e institucional de la UNAH.

La Universidad Nacional es pública y estatal y está atravesada por las políticas privatizadoras y excluyentes de los grupos de poder que dominan en Honduras. La institución como pieza clave en beneficio del modelo de acumulación se puede entender en dos sentidos:

1. Dominar el recurso educativo como instrumentalización ideológico-cultural. La educación es un acto necesariamente político; sin embargo, las lógicas de las políticas impuestas desde la llamada IV Reforma Universitaria ${ }^{10}$ se han centrado en la tecnocratización del conocimiento y la dominación ideológica neoconservadora sobre los sectores sindicales, estudiantiles y docentes; ello ha coaccionado las posibilidades del pensamiento crítico.

2. El vínculo directo entre el gobierno de Honduras y algunas de las autoridades universitarias para implementar el modelo de acumulación neoliberal. En el caso de la UNAH, este vínculo se produce desde "la instrumentalización del que yo llamo positivismo institucionalizado, esta fórmula consta de una lógica arcaica e impositiva en laquerigelaexcesiva cuantificación del sistema administrativo electrónico con su dimensión materializada en el sistema burocrático" (Briceño Cerrato, 2017: 3), impuesto con abrupta violencia simbólica en las tres funciones académicas de docencia, investigación y vinculación, dirigido por el neoconservadurismo de los marcos legales establecidos en las normas académicas de la IV Reforma.

El control de las tres funciones académicas implica el control del conocimiento como acto político, y la consiguiente tecnocratización de éste lleva a salidas laborales de precarización e inestabilidad. De esta manera, y en consonancia con la flexibilidad laboral y la precarización del trabajo — sobre todo juvenil- a nivel mundial, la universidad estaría creando perfiles de trabajadores con escasos derechos laborales, precarizados y acostumbrados a una alta rotación, en consonancia con las características de la mano de obra que requieren las empresas privadas hondureñas.

Asimismo, las políticas gubernamentales y universitarias entre 2009 y 2017 han conducido a la aplicación de pagos de matrícula, laboratorios y materiales cada vez más altos, y también a la expulsión de alumnos con bajo rendimiento académico y a la exclusión de estudiantes a través de la denominada "prueba de aptitud académica". Esta prueba favorece a los grupos estudiantiles procedentes de colegios ubicados en sectores de clases medias y altas, lo que conduce a una segregación social que refuerza desigualdades generales en lugar de revertirlas o mitigarlas.

Estas medidas excluyentes tomadas en una coyuntura de gran movilización social, sobre todo juvenil, han sido el detonante de las insurgencias estudiantiles desde 2013. Las contradicciones entre profesores, autoridades y estudiantes en el gobierno universitario, junto con los reclamos por mayor representación estudiantil en los procesos de toma de decisiones, fueron dos de los condicionantes de surgimiento del autodenominado Movimiento Estudiantil Universitario (MEU) en 2015. Este movimiento, que nació a partir del MAU, lo integran 
las facultades, organizadas en asociaciones, ${ }^{11}$ en todos los centros regionales universitarios del país. Surgió como una alternativa contrahegemónica, con accionares políticos de carácter multidisciplinario, e incluyó eventualmente en sus agendas de acción política a movimientos organizados de indígenas, docentes, sindicatos y otras organizaciones políticas de la ciudadanía.

Las diversas juventudes agrupadas en el MEU han creado "espacios sociales de realización plena de la democracia y de interrelación de campos de conocimientos que contribuyen con la redefinición de un nuevo sistema universitario concomitantes con las tendencias de producción del conocimiento interdisciplinario que demanda la diversidad" (Briceño Jiménez, 2007: 3).

Por lo tanto, la constitución del MEU abrió posibilidades de cambio y mayores condiciones de democratización del espacio universitario. Esta democratización se concibe no sólo como una reforma de las formas de gobierno de la universidad, sino también como parte del proceso de vinculación con la sociedad, al denunciar a los gobiernos nacionales fraudulentos y represores. Así, el MEU planteaba que, democratizando la universidad, se podría lograr conmover las estructuras autoritarias y represivas del Estado del que la UNAH forma parte (MEU, 2017b: 3).

Entre las demandas estudiantiles figuran la derogación de las normas académicas excluyentes, enmarcadas en la llamada "IV Reforma Universitaria", el cese de la criminalización de la protesta estudiantil, la represión, y la anulación de los requerimientos fiscales y órdenes de captura, consideradas por las autoridades como "delitos de sedición". Asimismo, los estudiantes agrupados en el MEU exigen mejoras en la calidad educativa y en la infraestructura del campus universitario en todos sus centros regionales, a la vez que se oponen a la mercantilización de la educación pública nacional.

En 2016 "las autoridades universitarias respondieron ante las demandas planteadas por los estudiantes con autoritarismo neoconservador y fascista, propiciando la violación de la autonomía en repetidas veces, en las que se extendieron órdenes de captura para 75 estudiantes" (Briceño Cerrato, 2017: 14). Como se mencionó, esto se enmarca en los procesos que caracterizaron la historia reciente hondureña, signada por violaciones a los derechos humanos de los estudiantes, que incluyen represiones, judicializaciones y estigmatizaciones que llegaban a su expulsión de la institución y hasta a su eliminación física.

Como parte de la reacción de las autoridades universitarias y nacionales a la movilización estudiantil, a mediados de 2017 tres estudiantes estuvieron a punto de ir a la cárcel. Según el Informe sobre el Movimiento Estudiantil Universitario, "el 7 de junio de 2017, los Sres. Cesario Padilla, Moisés Cáceres y Sergio Ulloa, fueron declarados culpables por un Tribunal de Sentencia de la Corte Suprema de Justicia (CSJ) del supuesto delito de usurpación" (MEU, 2017b: 17). Esto significó que fueron apartados de la vida universitaria y que se les impidió su matriculación trimestral. Sin embargo, el 19 de abril de 2018 la Corte Suprema de Justicia declaró inconstitucional el proceso a estos estudiantes y los reintegró a la vida universitaria como alumnos regulares (Conexión, 2018: 1).

Por su parte, la tenacidad del movimiento estudiantil en la búsqueda de conciliación y de diálogo ha sido constante aun después de los procesos judiciales y de los desalojos con militares y policías, en los que se violó en repetidas veces la autonomía universitaria. Una muestra de las políticas represivas que ganaron espacio en la UNAH es la evolución de la ejecución del presupuesto universitario desagregado "por grupo y objeto del gasto", donde consta el aumento constante del presupuesto en "equipos de seguridad y policía" (UNAH, 2017).

A inicios de septiembre de 2017 el conflicto se recrudeció y el gobierno universitario respondió en forma autoritaria, cediendo una vez más al ingreso de las fuerzas de seguridad y militares al Campus Universitario de Tegucigalpa. En el informe del MEU (2017b) sobre crisis institucional, derechos humanos y problemática general, se señala que hubo un desalojo violento por parte de más de quinientos efectivos de la Policía Nacional de los estudiantes que se estaban 
manifestando. Estas acciones de persecución y represión dieron como resultado el hostigamiento judicial de 37 defensores de derechos humanos, veinte estudiantes expulsados de la institución por un período de cinco años y más de veinte estudiantes judicializados. También se señala que de 2014 a junio de 2017 hubo un total de "234 procesos de criminalización de la protesta y discurso estatal violento" (MEU, 2017b). Estas acciones de represión evidencian las crecientes oposiciones estudiantiles a las autoridades que ejercen el gobierno universitario y que están vinculadas a las políticas represivas del gobierno de Honduras.

A inicios de 2018 las movilizaciones del MEU siguen en pie, así como las dinámicas de conflictividad y el antagonismo, que expresan una crisis hegemónica y de legitimación del gobierno universitario. Desde las carreras, facultades, centros regionales y organizaciones de base, el movimiento ha realizado acciones con pretensiones holísticas y multidisciplinarias a través de estrategias artísticas creativas que buscan democratizar los conocimientos y visibilizar las protestas desde otros formatos. Esta persistencia de las acciones públicas por parte de los estudiantes universitarios no tiene precedentes en la historia reciente del movimiento estudiantil hondureño.

Asimismo, el MEU ha demostrado iniciativa y vocación propositiva con la creación de herramientas como la Propuestapara una nueva reforma académica (2017c), en la que se desarrollan propuestas alternativas de estructuración académica, de gestión del conocimiento y cultural, de desarrollo curricular, internacionalización y movilidad académica, de movilidad educativa y del marco político legal de los estudiantes y los docentes en cuanto a su participación en el gobierno universitario. La persistente efervescencia movilizadora y la difusión de las propuestas alternativas generadas desde el MEU han posibilitado que la organización no sólo se mantenga en el tiempo, sino que crezca y se consolide integrando a grupos de estudiantes cada vez más numerosos. En este sentido, los universitarios que no forman parte de la militancia organizada han empatizado con las demandas y se han sumado en acciones cotidianas a las protestas del movimiento estudiantil. Estas numerosas adhesiones se manifestaron en prácticas de protesta como plantones, actos de difusión informativa y presentaciones artísticas. Así, los estudiantes universitarios no organizados en el MEU experimentaron procesos de politización que los acercaron a las movilizaciones y al activismo universitario.

Como parte del desarrollo del MEU se realizó en abril de 2017 el Congreso Nacional de Estudiantes Universitarios Eduardo Becerra Lanza, en el que confluyeron las diversas propuestas del movimiento. En este encuentro, el MEU y la Facultad de Ingeniería (2017d) expusieron la Propuesta para la restauración del Gobierno Estudiantil Universitario, en la que se presentaron alternativas, objetivos y estrategias para que las elecciones universitarias fueran más participativas y se considerara a los estudiantes. Estas acciones políticas, que incluyeron propuestas académicas y científicas elaboradas en conjunto con algunos sectores docentes, fueron espacios de confluencia intergeneracional que también sirvieron como expresiones de crítica al adultocentrismo que hegemoniza las interpretaciones de estos procesos. Estas acciones se desplegaron en una doble vía: por un lado, se tomaron los principales centros universitarios y, por el otro, se elaboró un anteproyecto de ley con orientaciones equitativas, inclusivas y democráticas que apuntaban a revertir las políticas regresivas a nivel universitario y nacional. Según este anteproyecto: "la comunidad universitaria no ha encontrado solución alguna a la crisis por medio de las vías institucionales internas, por lo que en esta ocasión nos vemos en la necesidad de acudir al Poder Legislativo" (MEU, 2017a: 2). De esta manera, frente a las actitudes de las autoridades universitarias, que eran interpretadas como intransigentes y autoritarias por el movimiento estudiantil, el anteproyecto de ley fue presentado directamente al Congreso Nacional. Para algunos sectores críticos del MEU esto significaba habilitar una intervención gubernamental directa en la vida universitaria a través de la representación parlamentaria del partido de gobierno, el Nacional.

Uno de los elementos centrales de la propuesta de ley fue la creación de una comisión amplia y participativa 
compuesta por docentes, trabajadores administrativos y estudiantes que velase por "la restauración de la participación estudiantil legítima y legal en los órganos de gobierno universitario; restablecer todos los órganos de gobierno de la UNAH, generar el relevo de las actuales autoridades universitarias, proteger y promover los derechos fundamentales de los estudiantes" (CriterioHn, 2017: 1). La respuesta del Congreso Nacional fue la revisión del anteproyecto de ley y la devolución del mismo al MEU con rotundas modificaciones que eliminaban las propuestas de participación equitativa de todos los sectores de la universidad en su gobierno $y$, por ende, no consideraban la democratización que buscaba por el movimiento estudiantil.

El autoritarismo del gobierno presidido por Juan Orlando Hernández y el control de las instituciones legislativas y judiciales, sumado al deterioro del diálogo entre estudiantes y autoridades universitarias y a algunas actitudes intolerantes por parte del gobierno de la universidad, complejizaron el escenario para los estudiantes. Sin embargo, las movilizaciones estudiantiles continuaron a través de expresiones culturales y estéticas o de performances de grupos afrodescendientes, de jóvenes indígenas, de grupos feministas, de diversidad sexual y de distintas organizaciones juveniles consideradas de izquierda. De esta manera, la movilización estudiantil en el espacio público continuó sosteniendo las expectativas de democratizar - y habitar - los espacios educativos y sociales en general.

\section{Crisis del sistema político y corrupción gubernamental como detonantes de las protestas juveniles entre 2009 y 2017}

Las actuales expresiones de resistencia estudiantil en Honduras encuentran algunas de sus raíces en la historia reciente del país, a partir de los ciclos de protesta que atraviesan tanto distintas zonas urbanas y rurales, así como en la historia de otros países del triángulo norte centroamericano, sobre todo de Guatemala.

Como se mencionó, en 2014 los procesos electorales que llevaron a la asunción de Juan Orlando Hernández como presidente tuvieron cuestionamientos de fraude y manipulación. Antes, el golpe de Estado de junio de 2009 que derrocó a Manuel Zelaya, quien había sido electo por voto popular en 2006, y el descontento popular ante las gestiones de gobierno posteriores, contribuyeron a la emergencia tanto de acciones de protesta callejera, como de resistencias organizadas alrededor del Frente Nacional de Resistencia Popular (FNRP, cercano al presidente depuesto) y de nuevos partidos políticos que desafiaron el bipartidismo imperante por décadas en Honduras.

Entre los grupos políticos que surgieron tras 2009 destacan el Partido Anti Corrupción, el Frente Amplio Electoral en Resistencia, la Alianza Patriótica y el Partido Libre. ${ }^{12}$ En las elecciones de 2013 este último partido obtuvo el " $28.78 \%$ de los votos, en relación al $36.82 \%$ de los votos por parte de Juan Orlando Hernández" (TSE, 2013), por lo que perdió en la contienda, que estuvo rodeada de acusaciones de fraude y de parcialidad de las autoridades electorales.

En efecto, las tendencias marcaban que el Partido Libre sería el ganador de los comicios, pero las irregularidades en los procesos de escrutinio y la manipulación de las elecciones, hechos fuertemente denunciados por los observadores internacionales, hicieron que el resultado fuese otro, y el Partido Nacional, continuador de los grupos que llegaron al poder tras el golpe de 2009, se impuso. En un informe poselectoral, el Partido Libre demostró que en el:

12\% de las urnas se denota una inflación de resultados, dado que el promedio de votación en cada MER es del $61 \%$ y en este bloque de urnas (12\%) la votación es mayor de $70 \%$, en tanto el conteo da $85 \%$ a favor de Juan Orlando Hernández, candidato presidencial por el Partido Nacional (Libre, 2013: 5). ${ }^{13}$

El descontento social era creciente, lo que provocó movilizaciones y protestas en diversos espacios en las que se vieron involucrados grupos sociales heterogéneos, entre los cuales destacaron las poblaciones juveniles. A finales de 2013, las protestas contra el fraude electoral que llevó a Juan Orlando Hernández a la presidencia se 
expresaron en la UNAH, que se convirtió en el campo de acciones colectivas que tuvieron gran visibilidad y difusión en redes sociales digitales. Entre fines de 2013 e inicios de 2014 se produjeron diversas manifestaciones en el campus universitario. La convocatoria tenía como principal demanda el esclarecimiento de los procesos electorales generales y constituyó el inicio de demostraciones en contra del gobierno y, sobre todo, de cuestionamientos públicos a la figura del presidente, a quien consideraban electo de forma irregular.

Este movimiento se identificó como "Movimiento de Camisetas Negras Anti-JOH". ${ }^{14}$ Este grupo estuvo compuesto tanto por las juventudes universitarias que militaban en organizaciones de la UNAH, entre las que desatacaba el MAU, como por jóvenes universitarios no militantes. Los "Camisetas Negras Anti-JOH" realizaron acciones públicas de protesta y fueron parte importante del emerger posterior de contundentes movilizaciones que desbordaron el espacio universitario.

En 2015 se revelaron en la prensa casos de corrupción sucedidos en el Instituto Hondureño de Seguridad Social (IHSS)..$^{15}$ En efecto, en 2013 el IHSS era presidido por Mario Zelaya, a quien, junto con un grupo de testaferros e integrantes del Partido Nacional, se le comprobó el robo de "unos 7.000 millones de lempiras, unos 335 millones de dólares" (Orellana, 2014). Según fuentes de prensa gráfica, como consecuencia de este desfalco a las arcas públicas "han muerto más de 2.880 personas por falta de atención sanitaria" (Criterio.hn, 2015). Esta situación de corrupción abierta y degradación de las políticas sociales fue el principal detonante de una nueva ola de movilización desencadenada a mediados del año 2015, que también encontró en las juventudes universitarias a sus principales protagonistas.

El movimiento que emergió de este descontento ciudadano se conoció como Movimiento de Antorchas. Entre sus principales demandas figuraron el esclarecimiento del latrocinio al IHSS, la solicitud de justicia para la resolución del caso y la renuncia del presidente Juan Orlando Hernández. Esta nueva ola de movilizaciones, que podría analizarse en el marco de un ciclo de protestas que comenzó en 2009, perduró por varios meses y tuvo fuertes impactos tanto en el gobierno, como en los procesos políticos y sociales de ese momento y posteriores.

La mayoría de los jóvenes que impulsaron y sostuvieron esas movilizaciones se autoidentificaban como "indignados", y vinculaban sus acciones con la oleada mundial de movimientos de indignados en distintos países. Asimismo, los reclamos principales de ese momento, que continuaron movilizando y aglutinando a sectores diversos en los años posteriores, eran la salida del presidente - "Fuera JOH" - y la instalación en Honduras de una Comisión Internacional contra la Impunidad, similar a la que bajo el mismo nombre se formó en Guatemala. ${ }^{16}$ Estas protestas incluyeron una huelga de hambre que un grupo de jóvenes de diversos sectores - estudiantiles, campesinos, populares - emprendió durante varios días, ocupando un espacio público en la zona céntrica de Tegucigalpa donde se encuentran varios de los más importantes edificios de gobierno.

Esta huelga de hambre protagonizada por jóvenes identificados como indignados fue el epicentro de grandes movilizaciones sociales que ocuparon las principales calles de Tegucigalpa. En estas acciones políticas la dimensión estética y expresiva era parte importante de la escenificación pública. Así, máscaras, muñecos, camisetas serigrafiadas, banners, carteles y pancartas constituyeron los símbolos de estas protestas para expresar dos procesos: por un lado, el de carnavalización y culturización de la participación política juvenil que señalaron autores como Reguillo (2003) para otras situaciones, y, por el otro, las disputas por el uso, la apropiación y la producción de los espacios públicos, tema que fue trabajado en otras coyunturas urbanas por Vommaro (2015a) como parte del proceso de territorialización de la política generacionalmente configurada.

La ocupación delespacio público para movilizaciones populares se produce en Honduras en otras situaciones vinculadas con festividades patrias o efemérides, en las cuales grandes grupos sociales salen a la calle y marchan respetando formas rígidas y ritualizadas que se reproducen año tras año. Sin embargo, cuando se trata 
de una acción de protesta protagonizada por juventudes movilizadas, estas ocupaciones del espacio público se llevan a cabo de manera más dinámica, menos formal y sin ceñirse a pautas dadas; es decir, produciendo modalidades de apropiación de las calles en las que las corporalidades se despliegan con otras lógicas surgidas de los propios procesos de politización.

En cuanto a las consignas coreadas en las movilizaciones, el hartazgo ante la corrupción y el autoritarismo que se vive en Honduras desde hace años, hizo que la pregunta generalizada que se formulaba en voz alta: “icuál es la ruta?", se respondiera con una frase contundente: "sacar a este hijueputa", seguida por un "fuera JOH".

De esta manera, el movimiento de 2015 tuvo gran incidencia en la consolidación de la organización juvenil universitaria que ya se venía gestando en años anteriores, vinculada con otras movilizaciones masivas sucedidas en otros países de América Latina. ${ }^{17}$

\section{Reflexiones finales}

Para concluir este artículo presentamos algunos comentarios que pueden ser también una guía para continuar trabajando los temas aquí analizados:

- El autoritarismo y los procesos políticos antidemocráticos en Honduras reflejan una crisis generalizada de representación política que se expresa también en la descendente legitimidad del gobierno nacional y del gobierno universitario.

- Los procesos de resistencia juvenil universitaria involucran diversidades sociales, políticas e ideológicas como las de los pueblos originarios, campesinos, las organizaciones feministas, los grupos de diversidad sexual y las organizaciones de izquierda. Al mismo tiempo, presentan elementos comunes en los discursos y en las prácticas. Entre ellos, la emergencia de estrategias de resistencia innovadoras, disruptivas, y con tendencias a la democratización y disputa de los espacios públicos.
- Las propuestas estudiantiles que avanzan en alternativas tanto políticas como académicas - presentación de un nuevo anteproyecto de ley - contribuyen a ampliar las bases de apoyo y a la masificación de los movimientos juveniles $y$, con ello, a la potenciación de sus estrategias de movilización y presentación en los espacios públicos.

- Las tendencias al debilitamiento y la degradación del régimen de gobierno tanto universitario como nacional presentan una oportunidad para consolidar los procesos políticos de emergencia de colectivos juveniles con propuestas creativas, alternativas y desafiantes de las prácticas adultocéntricas institucionalizadas. - Los valores y las prácticas vinculadas con la cooperación, la afectividad y la participación, sobre el individualismo, el pragmatismo y la representación, constituyen elementos comunes que hacen posible emprender acciones políticas articuladas en un marco de creciente diversidad de las juventudes hondureñas que emergen al calor de la visibilización de pluralidades étnicas, de género, territoriales y culturales.

- Antelaimposibilidad de obtener consensos para las políticas regresivas que el gobierno pretende imponer sobre la población se implementan medidas que buscan la imposición a través de la represión.

- El protagonismo creciente de las juventudes como grupo cultural, social y político posibilita el despliegue de procesos creativos, participativos y colaborativos que alimentan vínculos comunitarios y otras formas de relación social, potenciando la politización de los afectos e instituyendo el espacio educativo como ámbito de encuentro - y a la vez disputa - por lo público en tanto lo común.

- Las persistentes movilizaciones estudiantiles universitarias que se produjeron en Honduras luego de 2009 pueden interpretarse dentro de un ciclo de movilización juvenil más general, a nivel regional, que abarca países como Guatemala o Nicaragua. 


\section{Notas}

${ }^{1}$ El 28 de junio de 2009 tropas del ejército hondureño ocuparon la casa del presidente Manuel Zelaya —electo por voto popular en 2006 - y lo obligaron a dejar el país, lo que hizo viajando hacia Costa Rica. La excusa fue la ilegalidad de la llamada "cuarta urna" habilitada en las elecciones y el desacato al Poder Judicial. Esto abrió una ola de protestas y movilizaciones que se organizaron en el Frente Nacional de Resistencia Popular (FNRP). Para intentar legitimar el golpe de Estado se realizaron una serie de elecciones en las que se sospechó que hubo fraude y que dieron lugar a gobiernos autoritarios.

${ }^{2}$ Con esta noción retomada de Weber (1973), los autores explican el proceso de apropiación de recursos públicos por parte de una élite gobernante, en detrimento de las posibilidades de los grupos sociales más pobres.

${ }^{3}$ Si bien nos inclinamos por una definición de juventud que supere las delimitaciones etarias, demográficas y biológicas y tome en cuenta las dimensiones generacionales incorporando elementos de producción social histórica y cultural, para los fines estadísticos tomaremos el recorte convencional que se utiliza para el caso de Honduras y que define como jóvenes a las personas de entre 15 y 29 años de edad (INE, 2017).

${ }^{4}$ La pobreza total para Honduras en 2016 era del 61\%, según el INE (2017).

${ }^{5}$ La UNAH es la única universidad pública en Honduras que cuenta, además de su sede principal en Tegucigalpa, con ocho centros regionales en diversas regiones del país.

6 Si bien consideramos importantes y estimulantes estos análisis, aquí no profundizaremos en ellos por cuestiones de espacio y para enfocarnos en los objetivos del artículo.

${ }^{7}$ Es en esta época cuando se producen los primeros textos que abordan la juventud en tanto sujeto social y político desde la noción de generación, que será retomada en estos últimos años. Para ampliar esto, ver, por ejemplo, a Mannheim (1993 [1928]) y Ortega y Gasset (1996 [1928]). También en la década de 1920 se produjeron textos de autores como José Carlos Mariátegui (en Perú) y Julio Antonio Mella (en Cuba), ambos influidos por el movimiento reformista de 1918.
${ }^{8}$ Entre muchos otros trabajos interesantes sobre el movimiento estudiantil en la actualidad, destacan los de Galindo y Acosta (2011) sobre movimiento universitario en Colombia; Óscar Aguilera (2011 y 2012), Grez Toso (2012) y Raúl Zarzuri (2011) sobre movilizaciones secundarias y universitarias en Chile; y Núñez (2013), Enrique (2010), Larrondo (2012), Carli (2012) y Fernández Plastino (2010) para el caso argentino.

${ }^{9}$ Por politización entendemos el proceso por el cual las dimensiones personales, subjetivas y cotidianas de la vida individual y social devienen políticas y se vuelven parte del espacio público y de las cuestiones comunes. Es decir, un proceso en el que ciertas prácticas terminan siendo políticas al expresar antagonismos sociales situados en una coyuntura socio-histórico-cultural singular (Bonvillani et al., 2010).

${ }^{10}$ La denominada IV Reforma de la UNAH inició en 2005 tras la aprobación de la Ley Orgánica de la Universidad Nacional Autónoma de Honduras en diciembre de 2004. El objetivo era adecuar la UNAH a las "demandas de la sociedad hondureña" para "hacer frente a los grandes problemas y aspiraciones de los hondureños" (Ley Orgánica de la Universidad Nacional Autónoma de Honduras, 2004). Muchas voces críticas señalaron que esta Reforma sirvió para realizar una concentración de poder en manos de las autoridades universitarias y que no logró producir una ampliación en la participación de los diferentes sectores en el gobierno de la Universidad. A raíz de estos análisis, algunos sectores denominaron esta Reforma como "Contra Reforma de la UNAH" (Briceño Cerrato, 2017).

${ }^{11}$ Las asociaciones de estudiantes son la base social del movimiento. Las demandas son fundamentalmente de estructuración institucional de nuevos espacios democráticos.

${ }^{12}$ El Partido Libre surgió como el brazo político del Frente Nacional de Resistencia Popular (FNRP), principal e inmediata oposición al golpe de Estado de 2009. En el proceso electoral de 2013 la candidata a la presidencia fue Xiomara Castro de Zelaya, esposa del depuesto presidente José Manuel Zelaya.

${ }^{13}$ Este informe fue realizado después de las elecciones; además de contener fuertes cuestionamientos legales al 
proceso electoral, contiene críticas al escrutinio desde las organizaciones internacionales que vigilaron el proceso.

${ }^{14} \mathrm{JOH}$ es la manera en que se nombra al presidente Juan Orlando Hernández a nivel popular; se usan las siglas de su nombre como apelativo en consignas y cantos de protesta.

${ }^{15}$ Ente encargado de brindar cobertura médica a los trabajadores hondureños, creado en 1959 mediante la Ley de Seguridad Social en Honduras.

${ }^{16} \mathrm{La}$ CICIG fue creada en 2006 mediante pactos entre el Gobierno de Guatemala y las Naciones Unidas.

${ }^{17}$ Estas movilizaciones se produjeron de manera singular en cada país; para el caso de Chile, entre 2006 y 2012 con el movimiento de Los Pingüinos; en Colombia fueron protagonizadas por la Mesa Amplia Nacional Estudiantil (MANE) en 2011 y el Movimiento \#YoSoyl32 se expandió en México en 2012. Para ampliar, ver Vommaro (2013 y 2015a).

\section{Referencias}

Aguilera, Óscar (2011). “Acontecimiento y acción colectiva juvenil. El antes, durante y después de la rebelión de los estudiantes chilenos en el 2006". En Propuesta Educativa, 35(1): 11-26.

Aguilera, Óscar (2012). "Repertorios y ciclos de movilización juvenil en Chile (2000-2012)”. En Utopía y Praxis Latinoamericana, 17(57): 101-108.

Alcántara Sáez, Manuel y Juan Manuel Ibeas Miguel (2001). Colombia ante los retos del siglo XXI. Desarrollo, Democracia y Paz. Salamanca: Ediciones Universidad de Salamanca.

Badiou, Alan (2000). Movimiento social y representación política. Buenos Aires: Instituto de Formación de la CTA.

Banco Mundial (2017). "Honduras, panorama general". En El Banco Mundial en Honduras, 7 de abril. Disponible en: http://www.bancomundial.org/es/country/honduras/ overview $\not 1$.

Bonvillani, Andrea, Alicia Palermo, Melina Vázquezy Pablo Vommaro (2010). "Del Cordobazo al kirchnerismo. Una lectura crítica acerca de los períodos, temáticas y perspectivas en los estudios sobre juventudes y participación política en la Argentina”. En S. Alvarado y Pablo Vommaro (eds.), Jóvenes, cultura y política en América Latina: algunos trayectos de sus relaciones, experiencias y lecturas (1960-2000). Buenos Aires: CLACSO, Homo Sapiens, pp. 21-54.

Briceño Cerrato, Galel (2017). Escenarios de expulsión sistémica y conflictividad política: Universidad Nacional Autónoma de Honduras. Tegucigalpa: s.d, 20 de marzo.

Briceño Jiménez, Roberto (2007). "La Universidad: entre las fuerzas neoconservadoras y la fuerza emergente de la transformación democrática y emancipadora" [mensaje en un blog]. Tegucigalpa. Disponible en: http://libertaddeepresionhn.blogspot.mx/.

Carli, Sandra (2012). El estudiante universitario. Hacia una historia del presente de la educación pública. Buenos Aires: Siglo XXI Editores.

Castro-Gómez, Santiago (2010). "Siglo XVIII: el nacimiento de la biopolítica". En Tabula Rasa 12: 31-45.

CEPAL y UNFPA (2014). Invertir en juventud en América Latina y el Caribe: un imperativo de derechos e inclusión. Panamá: CEPAL, UNFPA.

Criterio.hn (2015). "Alrededor de tres mil personas han muerto por desfalco al IHSS". En criterio.hn, 28 de mayo. Nacionales. Disponible en: https:/criterio. $\mathrm{hn} / 2015 / 05 / 28 /$ alrededor-de-tres-mil-personas-hanmuerto-por-desfalco-al-ihss/.

Criterio.hn (2017). "En masiva movilización MEU entrega propuesta al Congreso Nacional". En criterio.hn, 19 de julio. Disponible en: https:/criterio.hn/2017/07/19/ masiva-movilizacion-meu-entrega-propuesta-alcongreso-nacional/.

Conexihon.hn (2018). "Declaran inconstitucional reglamento estudiantil en la UNAH”. En Conexión.hn, 19 de abril. Disponible en: http://www.conexihon.hn/index.php/ libertad-de-expresion/644-declaran-inconstitucionalreglamento-estudiantil-de-la-unah

Enrique, Iara (2011). “La participación estudiantil en la escuela secundaria en la Argentina. Reconstrucción del conflicto en torno al protagonismo político de los jóvenes". Tesis de Maestría en Políticas Sociales. Facultad de Ciencias Sociales, Universidad de Buenos Aires.

Fernández Plastino, Alejandro (2010). "Movimiento estudiantil: de la política a lo político; de las 
organizaciones a la multitud". En Primeras Jornadas Debates Actuales de la Teoría Politica Contemporánea, Buenos Aires.

Grez Toso, Sergio (2012). "Chile 2012: el movimiento estudiantil en la encrucijada". En Le Monde Diplomatique, enero-febrero de 2012. Chile.

INE (Instituto Nacional de Estadística de Honduras) (2017). Hogares en condición de pobreza 2016. Tegucigalpa: INE.

IUDPAS (Instituto Universitario de Democracia, Paz y Seguridad) (2016). Boletín especial sobre homicidios en estudiantes. Tegucigalpa: Observatorio Nacional de la Violencia.

Jelin, Elizabeth (1989). "Los movimientos sociales en la Argentina contemporánea: una introducción a su estudio". En Elizabeth Jelin (comp.), Los nuevos movimientos sociales. Mujeres. Rock nacional. Derechos humanos. Obreros. Barrios. Buenos Aires: Centro Editor de América Latina, pp. 36-40.

Larrondo, M. (2012). “iNuevos alumnos? La construcción de la identidad escolar en nuevos dispositivos de educación media. Un estudio de casos en contextos de pobreza de la Ciudad de Buenos Aires". En Pensamiento Educativo, 49(2): 18-31. Santiago de Chile.

Libre (2013). Informe ejecutivo preliminar: elecciones generales 2013. Tegucigalpa: Partido Libertad y Refundación.

MEU (Movimiento Estudiantil Universitario) (2017a). Ante Proyecto de Ley. Tegucigalpa: MEU.

MEU (Movimiento Estudiantil Universitario) (2017b). Informe sobre el Movimiento Estudiantil Universitario: Crisis institucional, derechos humanos y problemática general. Tegucigalpa: MEU.

MEU (Movimiento Estudiantil Universitario) (2017c). Propuesta para la elaboración de nuevas normas académicas. Tegucigalpa: MEU.

MEU (Movimiento Estudiantil Universitario) y Facultad de Ingeniería (2017d ). Propuesta para la restauración del gobierno estudiantil universitario. Tegucigalpa: MEU.

Nateras, Alfredo (2015). "El aniquilamiento identitario en Centroamérica”. En José Manuel Valenzuela (coord.), Juvenicidio. Ayotzinapa y las vidas precarias en América Latina y España. Barcelona: Ned, pp. 99-132.

Núñez, Pedro (2013). La política en la escuela. Jóvenes, justicia y derechos en el espacio escolar. Buenos Aires: La Crujía.
Orellana, Dunia (2014). "Mario Zelaya capturado por desfalco al IHSS". En La Prensa, 11 de septiembre. Disponible en: http://www.laprensa.hn/sucesos/ policiales/746039-98/mario-zelaya-capturado-pordesfalco-al-ihss.

Pena, Gustavo (2014). "Unos 800,000 jóvenes ni estudian ni trabajan en Honduras". En El Heraldo, 7 de abril. Disponible en: http://www.elheraldo. hn/alfrente/564927-209/unos-800000-jovenes-niestudian-ni-trabajan-en-honduras (consultado el 7 de abril de 2017).

PNUD (2015). Panorama general. Informe sobre desarrollo humano 2015. Trabajo al servicio del desarrollo humano. Nueva York: PNUD.

Reguillo, Rossana (2003). Emergencia de culturas juveniles. Estrategias del desencanto. Buenos Aires: Norma.

Rodríguez, Ernesto (2012). Movimientos juveniles en América Latina: entre la tradición y la innovación. Montevideo: CELAJU, UNESCO.

Schütz, Alfred (1993). La construcción significativa del mundo social. Introducción a la sociología comprensiva. Barcelona: Paidós.

Sosa, Eugenio (2013). Dinámica de la protesta social en Honduras. Tegucigalpa: Guaymuras.

Sosa, Eugenio (2014). "Honduras: entre criminalidad, enfrentamiento mediático, protesta social y resultados electorales cuestionados". En Revista de Ciencia Política, 34(1): 203-219. Santiago de Chile.

Tapia, Luis (2008). Política salvaje. La Paz: CLACSO, Comuna.

TSE (Tribunal Supremo Electoral) (2013). "Resultados electorales". Dsponibleen:http://siede.tse.hn/escrutinio/ index.php (consultado el 20 de septiembre de 2017).

Tünnermann Bernheim, Carlos (2008). Noventa años de la Reforma Universitaria de Córdoba (1918-2008). Buenos Aires: CLACSO.

UNAH (2017). "Ejecución del presupuesto de egresos por grupo y objeto del gasto”. En Portal de Transparencia de la UNAH. Tegucigalpa: UNAH. Disponible en: https://transparencia.unah.edu.hn.

Valenzuela Arce, José Manuel (2015). Juvenicidio. Ayotzinapa y las vidas precarias en América Latina y España. Barcelona: Ned. 
Vommaro, Pablo (2013). "Las relaciones entrejuventudes y políticas en la América Latina contemporánea: una aproximación desde los movimientos estudiantiles". En Sociedad, 32: 127-144.

Vommaro, Pablo (2015a). Juventudes y políticas en la Argentina y en América Latina. Tendencias, conflictos y desafíos. Buenos Aires: Grupo Editor Universitario.

Vommaro, Pablo (2015b). "Movilizaciones juveniles en América Latina actual: hacia las configuraciones generacionales de la política”. En Controversias y Concurrencias Latinoamericanas, 7(11): 25-54.
Weber, Max (1973) Ensayo sobre metodología sociológica. Buenos Aires: Amorrortu.

Wiarda, Howard (1997). "Determinantes históricos del Estado latinoamericano: la tradición burocrática-patrimonialista, el corporativismo, el centralismo y el autoritarismo". En Menno Vellinga, El cambio del papel del Estado en América Latina. México: Siglo XXI Editores, pp. 45-73.

Zarzuri, Raúl (2011). Jóvenes, participación y construcción de nuevas ciudadanías. Santiago de Chile: CESC. 